\title{
A Case Report of Bilateral Trigeminal Neuralgia Combined with Bilateral Glossopharyngeal Neuralgia
}

\author{
WEI Sheng Cheng ${ }^{1 *}$ and Qu Chun Cheng ${ }^{2}$ \\ Department of Neurosurgery, Shandong University, China \\ *Corresponding author: WEI Sheng Cheng, Department of Neurosurgery, Shandong University, China \\ Submission: January 23, 2019; Published: February 19, 2019
}

\section{Background}

The same patient coexisting multiple cranial nerve disorders is extremely rare, only about $1 \%$ of the data [1]. We treated a case of bilateral trigeminal neuralgia combined with bilateral glossopharyngeal neuralgia, and it had not been reported before. Now we report this case as follows.

\section{Case Presentation}

The patient was a 63-year-old-aged female, who experienced 7 years of bilateral cheek and oropharyngeal burning and stabbing pain. Touching the mouth and bilateral throat or swallowing could provoke the pain. Each episode of pain lasted a few seconds to a few minutes, and in severe cases could last for hours. Oral administration of carbamazepine and radiofrequency treatment before hospitalization had only poor effects. We diagnosed the patient as bilateral trigeminal neuralgia combined with bilateral glossopharyngeal neuralgia. After hospitalization, brain MRI examination was performed and did not reveal any occupational lesions. The bilateral pain of pharyngeal pain disappeared after the treatment of $1 \%$ butanocaine respectively, and this confirmed the diagnosis of bilateral glossopharyngeal neuralgia. The patient complained that the pain of right side was more serious than the other side. In 2015.05, a right trigeminal nerve microvascular decompression and glossopharyngeal nerve microvascular decompression was performed of posterior sigmoid sinus approach under general anesthesia. under a surgical microscope we saw the adhesion between the posterior cranial nerves and posterior inferior cerebellar artery, and the root entry zone of right trigeminal nerve was compressed by the right superior cerebellar artery. Respectively, Teflon cotton was placed between nerves and vessels to separate them. The pain of right facial and throat completely disappeared after this operation. The patient complained of mild cough when drinking water, and the symptoms disappeared 1 month later. 5months later, the patient experienced another microvascular decompression surgery of the trigeminal nerve and glossopharyngeal nerve of left side. The responsible blood vessel of left side glossopharyngeal neuralgia was posterior inferior cerebellar artery, and similarly with the right side, the adhesion between nerves and vessels was serious. The responsible blood vessel of left trigeminal neuralgia was the branch of the petrosal vein and the vascular loop of superior cerebellar artery. We severed the adhesions of the glossopharyngeal nerve with the arachnoid and the vessels using the microsurgery scissors, the teflon cotton was placed between glossopharyngeal nerve and the Posterior inferior cerebellar artery. After electrocoagulation cut off the branch of the petrosal vein, Teflon cotton was placed to separate the trigeminal nerve and superior cerebellar artery. Postoperative follow-up revealed that the bilateral pain was disappeared, and the effect of operations were good (Figures 1-4).

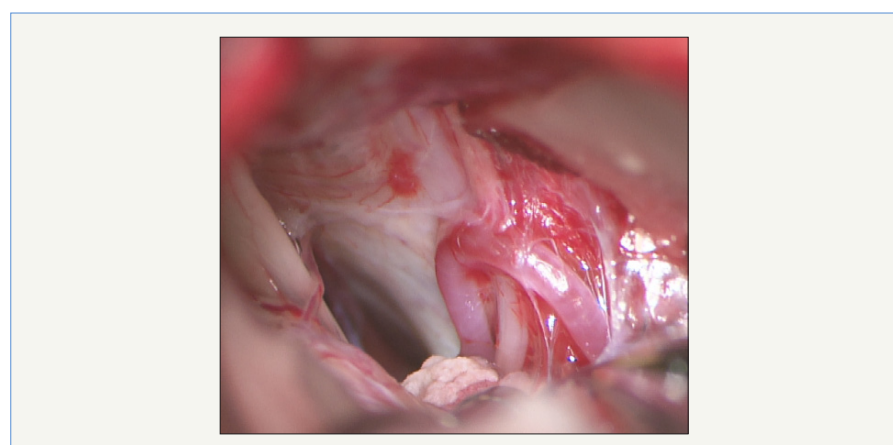

Figure 1: The root entry zone of right trigeminal nerve was compressed by the right superior cerebellar artery. (III, trigeminal nerve; A, superior cerebellar artery).

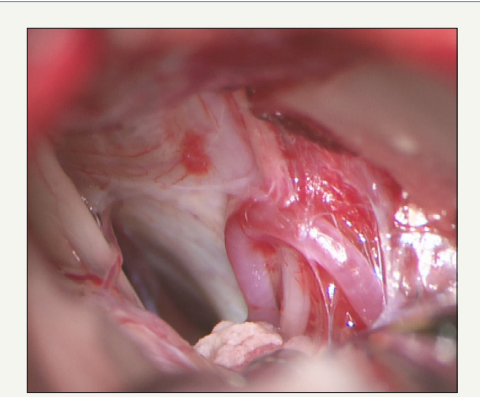

Figure 2: The adhesion between the posterior cranial nerves and posterior inferior cerebellar artery was severe, and the posterior inferior cerebellar artery was fixed (IX, glossopharyngeal nerve; $\mathrm{P}$, cerebellar inferior artery). 


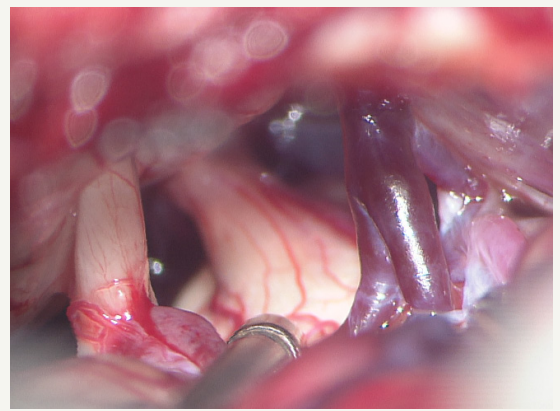

Figure 3: The compression to left trigeminal nerve root entry zone mainly come from the branch of petrosal vein (P), and the superior cerebellar artery(A) was also involved.

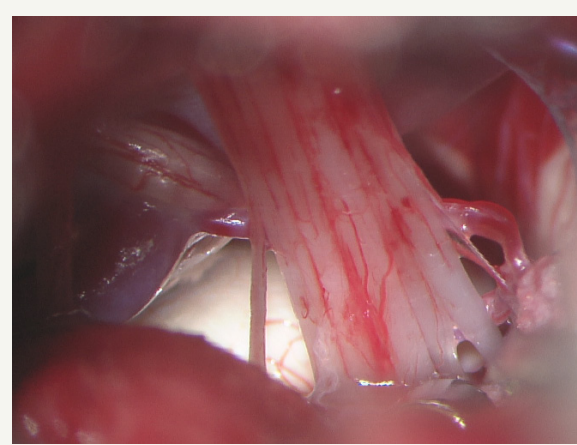

Figure 4: After the separation of adhesion, showing the posterior cranial nerves and posterior inferior cerebellar artery (X, glossopharyngeal nerve; ssIX vagus nerve)

\section{Discussion}

The incidence of Trigeminal neuralgia in china and abroad is $47.8 / 10$ million and $62.6 / 10$ million separately, more women than men, and the incidence rate can rise with age. The trigeminal neuralgia is more than this number in the population. bilateral trigeminal neuralgia is much more rare (0.6\%-5.3\%), usually one side after another, pain attack at the same time is even less. Glossopharyngeal neuralgia is extremely rare, characterized by pain on tonsil, pharynx, lingual and middle ear, and the pain is strictly limited to unilateral. Janneta [2] invented the concept of vascular compress REZ (root enter zoon, REZ, nerve root into the brain area) led to cranial nerve disease and through microvascular decompression succeeded to cure trigeminal neuralgia, Hemifacial Spasm and glossopharyngeal neuralgia. Since then, microvascular decompression (microvascular decompression, MVD) has gradually become the preferred treatment of primary trigeminal neuralgia and primary glossopharyngeal neuralgia. For the treatment of glossopharyngeal neuralgia, Taha [3] and Ferrante [4] advocated the best surgery way is microvascular decompression combine with glossopharyngeal nerve root incision, but few patients with postoperative complications of dysphagia, hoarseness and other complications. Now most scholars believe that simple microvascular decompression can reach $100 \%$ cure rate, and the incidence of cranial nerve injury may be further reduced along with the improvement of surgical technique. Sindou M [5] reported the cure rate of trigeminal nerve MVD was $91 \%$ in 420 cases, while glossopharyngeal neuralgia MVD cure rate was 100\% in 9 patients. Kondo [6] \& Sampson et al. [7] also hold the same view. Some scholars believe that the pathogenesis of glossopharyngeal neuralgia is due to compression of vertebral artery or inferior cerebellar artery and other abnormal vascular, along with the local thickening and adhesions of the arachnoid lead to glossopharyngeal nerve and vagus nerve root demyelination, so that a short circuit between the nerve fibers trigger the onset of pain [8]. Our This case also confirmed this point, around both side of glossopharyngeal nerve we saw severe arachnoid adhesions, while on both side of trigeminal nerve we did not find such adhesions, indicating that local arachnoid thickening and adhesions is Important pathogenic factor for glossopharyngeal neuralgia. The glossopharyngeal nerve enter into the craniotomy through jugular foramen, and part of the impulse can pass through the solitary nucleus to the vagus nerve dorsal nucleus, and some nerve fibers terminate in the trigeminal nerve spinal nucleus, so some stimulation of trigeminal neuralgia can simultaneously generate Pharyngeal neuralgia. This view can partly explain the causes of trigeminal neuralgia combine with glossopharyngeal neuralgia. In this case, bilateral trigeminal nerve and bilateral glossopharyngeal nerve all could be seen the compression of blood vessels, and MVDs were effective, this further verified the theory of vascular compression and the Security and effectiveness of MVD. The patient with bilateral trigeminal neuralgia combined with bilateral glossopharyngeal neuralgia at the same time, is extremely rare, and we have not found precedent report after information retrieval.

\section{References}

1. Zhang Li, Yu Yan Bing, Feng L (2004) Microvascular decompression therapy for multiple cranial nervous diseases. Chinese neurosurgery journal 20: 299-302.

2. Janneta PJ (1977) Observations on the etiology of trigeminal neuralgia hemifacial spasm acoustic nerve dysfunction and glossopharyngeal neuralgia. Definitive microsurgical treatment and results in 117 patients. Neurochirurgia Stuttg 20(5): 145-154]

3. Taha JM, Tew JM (1995) Long term results of surgical treatment of idiopathic neuralgias of the glossopharyngeal and vagal nerves. Neurosurgery 36(5): 926-931.

4. Ferrante L, Artico M, Nardacci B, Fraioli B, Cosentino F, et al. (1995) Glossopharyngeal neuralgia with cardiac syncope. Neurosurgery 36(1): 58-63.

5. Sindou M, Mertens P (1993) Microsurgical vascular decompression (MVD) in trigeminal and glossovagopharyngeal neuralgias. A twenty year experience. Acta Neurochir Suppl (Wien) 58: 168-170.

6. Kondo A (1998) Follow up results of using microvascular decompression for treatment of glossopharyngeal neuralgia. J Neurosurg 88(2): 221225.

7. Sampson JH, Grossi PM, Asaoka K, Fukushima T (2004) Microvascular decompression for glossopharyngeal neuralgia: longterm effectiveness and complication avoidance. Neuosurgery 54(4): 884-889.

8. Amthor KF, Eide PK (2003) Glossopharyngeal neuralgia. Tidsskr Nor Laegeforen 123(23): 3381-3383. 
Creative Commons Attribution 4.0 International License

For possible submissions Click Here

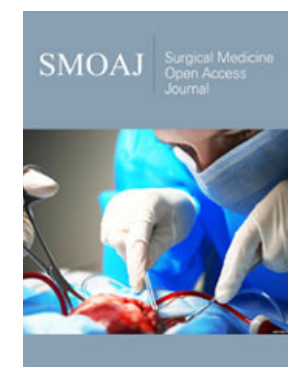

Surgicall Medicine Open Access Journal

\section{Benefits of Publishing with us}

- High-level peer review and editorial services

- Freely accessible online immediately upon publication

- Authors retain the copyright to their work

- Licensing it under a Creative Commons license

- Visibility through different online platforms 